\title{
LINEAR MIMO CONTROLLER DESIGN FOR AN INDUSTRIAL REACTIVE DISTILLATION COLUMN
}

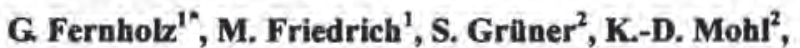 \\ A. Kienle ${ }^{3}$ and E.-D. Gilles ${ }^{2,3}$ \\ 'Bayer AG Process Technology, Process Control,51368 Leverkusen, Germany \\ ("Email: gregor.fernholz.gf@bayer-ag.de) \\ ${ }^{2}$ Institut für Systemdynamik und Regelungstechnik, University of Stuttgart, \\ 70550 Stuttgart, Germany \\ ${ }^{3}$ Max Planck Institut fïr Dynamik komplexer technischer Systeme, \\ 39120 Magdeburg, Germany
}

\begin{abstract}
The aim of this paper is to demonstrate the use of advanced linear controller design methods for an industrial scale reactive distillation column. The open-loop analysis of the process behavior indicates that linear control of the process is promising. Therefore, a control structure selection is made by an analysis of the physical behavior of the system and a RGA-analysis. Afterwards a frequency response approximation technique is used for the design of linear MIMO-controllers. Robustness of the closed loop is observed during the controller design. Finally, the controller is tested on the nonlinear process model. Copyright $(2001$ IFAC
\end{abstract}

Keywords: linear control, robust control, reactive distillation, continuation method.

\section{INTRODUCTION}

Control of distillation columns has been a research area now for several decades. Several excellent surveys in this area were published (e.g. Shinskey, 1984; Skogestad and Morari 1987; Luyben, 1992; and Skogestad, 1997). Since modern process control systems allow to implement more complex control structures, advanced controllers can be considered for industrial processes.

In order to investigate the benefits that can be gained from applying advanced process control to industrial scale applications, a reactive distillation process from the Bayer AG was chosen. The results of this work are presented in two parts. The first part, presented in this paper, deals with open-loop analysis, control structure selection, linear controller design, and robustness considerations. The second part by Grüner, et al. (2001) examines the nonlinear behavior and control of the process in more detail.

First, a short description of the process is given. A nonlinear process model is investigated by continuation methods to get first insight into the system's behavior. The first step in the controller design proce- dure is the selection of an adequate control structure. A sensitivity analysis of the temperature profile and of the product composition as well as a relative gain array (RGA) analysis are used to select favorable locations for the temperature sensors. The temperatures are used as controlled variables because on-line measurement of the compositions is not available at the plant. The next step in the design procedure is the calculation of a multivariable controller. The design method used here is a frequency response approximation procedure developed by Engell and Müller (1993). The influences of the desired bandwidth of the closed loop, of the orders of the controllers, and of the choices of the number of SISO controllers used in the MIMO controller (e.g. full MIMO controller or diagonal controller) on the controller performance and on the robustness are investigated. The controllers showing good performance during the controller design procedure are finally tested in simulations with the nonlinear process model.

\section{PROCESS DESCRIPTION}

The aim of the process is to produce a substance $\mathrm{E}$ by reactive distillation from the feed components $B$ and 
C. The simplified reaction scheme is given by equations (1) and (2):

$$
\begin{aligned}
& A+E \leftrightarrow B+D \\
& A+D \leftrightarrow B+C
\end{aligned}
$$

The scheme of the process is depicted in Fig. 1. The components $\mathrm{B}$ and $\mathrm{C}$ are fed to the column. The lower boiling component $\mathrm{A}$ is removed form the process via the head product. The desired higher boiling product $\mathrm{E}$ is recovered in the bottom product. The heat duty $Q$ supplied to the reboiler and the reflux ratio $R$ are used as manipulated variables for the controller design.

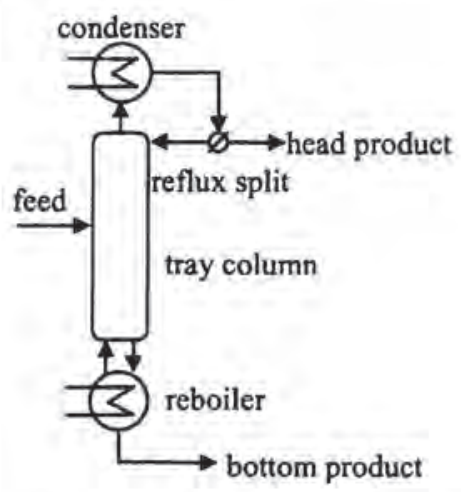

Fig. 1: Reactive distillation process scheme.

A nonlinear process model based on the concept of theoretical stages is derived from mass and energy balances. The non-ideal liquid phase behavior as well as the reaction kinetics are considered. The process model consist of about 250 differential and 4800 algebraic equations. This model is used for the generation of a linear system and for the nonlinear simulation.

All numerical values of process variables given in the sequel are meaningfully scaled and physical units are omitted to mask the information about the true operation of the process.

\section{NONLINEAR DYNAMICS}

The process behavior is investigated by continuation methods and stability analysis (Kienle et al. 1995). These methods give a very condensed information of the process behavior. Especially one can check for uniqueness and stability of steady state solutions. Usually it is no problem to stabilize an open loop unstable column with suitable control. However, in case of multiple steady states, special care has to be taken during column startup to get the column close to the desired steady state (Mohl et al. 1999). Note, that open loop instability and multiplicity of steady states are well known patterns of behavior in reactive distillation (see e. g. Mohl et al. 1999).
For the process considered here continuation of the steady states w.r.t. the manipulated variables shows that for the operating condition used in the real plant no multiple steady states occur. Further the stability analysis reveals that there is always a unique stable steady state. This result is shown exemplarily in Fig. 2 for the continuation of the heat duty, which is one of the principal operating parameters.
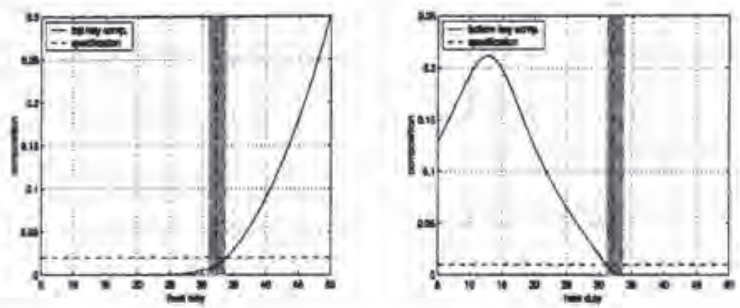

Fig. 2: Bifurcation diagram w.r.t. the heat duty

In Fig. 2 the solid lines show the composition of the key components in the top (comp. C, left) and bottom (comp. D, right) product. The broken lines give the specifications for the key components and the nominal operating value of the heat duty. The gray band indicates the in-spec operation of the plant. From that the gray band is rather narrow it can be concluded that a high performance controller is needed.

Further insight in the process dynamics can be obtained from column profiles as illustrated in Fig. 3. It was found that the column considered here is operated close to chemical equilibrium for both reactions. Using nonlinear wave propagation theory it can be shown, that under these conditions the column profiles of a reactive distillation column consists of NCNR-1 independent wave fronts in each column section (Gehrke et al. 1998, Kienle, 2000b). Here, NC and NR denote the number of components and reaction, respectively. After a disturbance some of these fronts - depending on the sign and size of the disturbance - are propagated through the column.

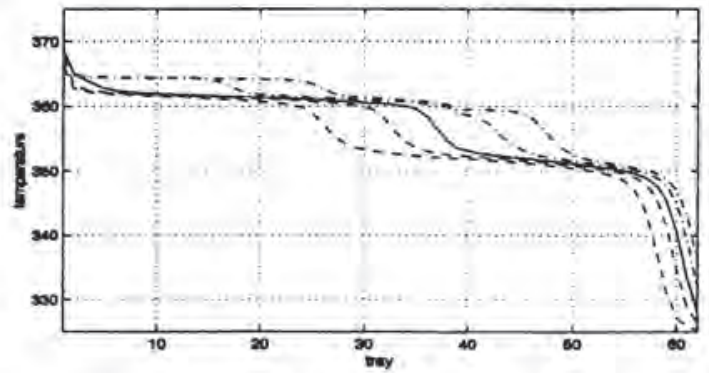

Fig. 3: Steady state temperature profiles for perturbation of the heat duty of $\mathrm{dQ}=+4 /+8$ (dash-dot), and $d Q=-4 /-8$ (dashed) from the given operating point.

This type of dynamic behavior is qualitatively similar to the nonreactive case with $\mathrm{NR}=0$ (Kienle, 2000a). From this point of view it can be expected that con- 
trol concepts from nonreactive distillation can be extended to reactive distillation columns. In particular, linear control seems promising to handle small disturbances close to the given operating point.

\section{CONTROL PROBLEM FORMULATION}

The main requirements that have to be satisfied by the controller are:

- The closed loop has to be stable.

- The controller has to be robust.

- Maximum bandwidth for the closed loop.

- Rejection of impurity increases due to disturbances

- observe bounds on the manipulated variables.

The aim of the controller design procedure is to satisfy as many requirements as possible. The nominal and robust stability and performance are checked by the $\mu$-conditions. The definition of the $\mu$-conditions and the mathematical derivation are beyond the scope of this paper but a detailed description can be found in Skogestad and Postlethwaite (1996). The uncertainties that are taken into consideration are caused by delays in the manipulated variables. Since the manipulated variables are themselves controlled by lower level controllers their speed of change is bounded. In the robust analysis framework this is treated as a diagonal input uncertainty.

\section{CONTROL STRUCTURE SELECTION}

The first step in the design procedure is to select an appropriate control structure. This includes the determination of the best locations of the temperature sensors that are used as controlled variables. A common strategy to locate the position of the temperature sensors is to calculate the temperature sensitivities (Moore, 1992).

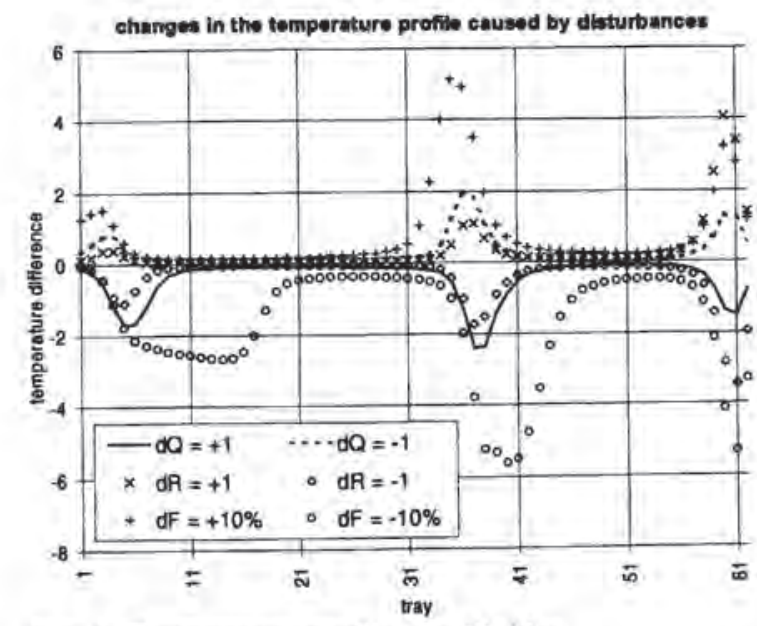

Fig. 4. Temperature profile sensitivities
The sensitivity of the temperature profile to changes in the reflux ratio of $d R= \pm 1$, in the heat duty of $\mathrm{dQ}= \pm 1$ and in the feed flow of $\mathrm{dF}= \pm 10 \%$ can be assessed from Fig. 4. The temperature profile shows significant changes in the upper (trays 55-62), the middle (trays 31-43) and the lower (trays 2-8) sections of the column. A good choice is to locate the sensors on trays 4,37 and 60 because there the responses are approximately symmetrical.

The next step in the control structure selection is an investigation of the relative gain array (RGA) and the frequency depended RGA-number. Therefore the nonlinear model is linearized at the nominal operating point. The system inputs are the heat duty and the reflux ratio, the system outputs the temperatures on trays 4,37 and 60 . The resulting state space model contains about 248 differential equations. For analysis and controller design purposes a low order linear model is more suitable and a reduction of the model order is necessary. The model reduction technique used here is based on a frequency response approximation and yields a model of order 25. Details on the method can be found in Fernholz et al. (2000). The comparison between the frequency responses of the high order and the reduced order model for the transmission path from the heat duty to the temperature on tray 4 in Fig. 5 shows an excellent correspondence. The accuracy of the approximations of the other transmission paths is comparable.
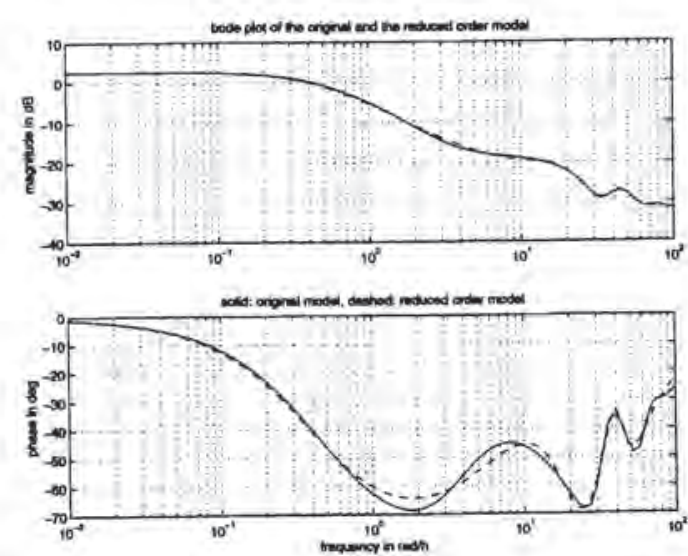

Fig. 5. Frequency responses of the transmission path heat duty to tray temperature 4 of the original linear model (solid) and the reduced model (dashed).

Since the system consists of three outputs and two inputs, three sets of $2 \times 2$ square system are possible. Therefore the steady state RGA (cf. Table 1) and the RGA-numbers (cf. Fig. 6) are calculated for all three sets using the reduced order model. The pairing rules (Skogestad and Postlethwaite, 1996) suggest that the temperature pairs $T_{4} / T_{60}$ and $T_{37} / T_{60}$ are preferable because both have RGA elements on the diagonal close to unity and low RGA-numbers over the entire frequency range. Thus the selection has to be made 
between the parings $T_{4} / T_{60}$ and $T_{37} / T_{60}$. Checking the influence of multivariable effects of model uncertainty by the minimized condition numbers does not lead to a decision: The minimized condition numbers lie between 1 and 3 over the entire frequency range in both cases.
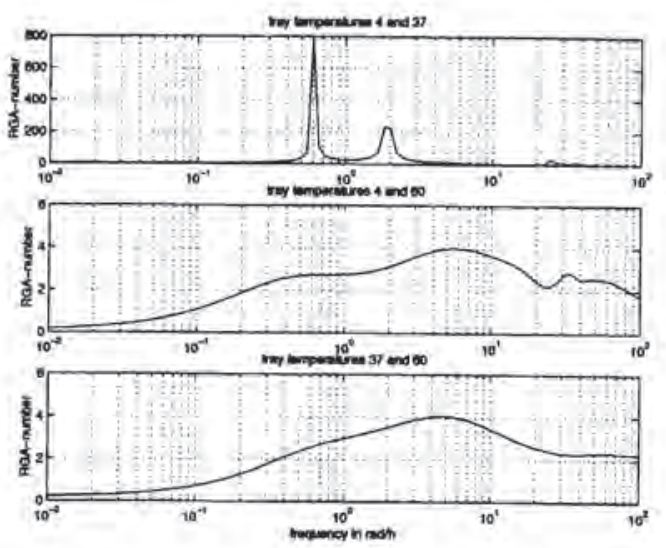

Fig. 6. RGA-numbers for the three sets of $2 \times 2$ systems.

Table 1. RGAs for the three sets of $2 \times 2$ systems.

\begin{tabular}{cccccc}
\hline \multicolumn{2}{c}{ trays 4 and 37} & \multicolumn{2}{c}{ trays 4 and 60} & \multicolumn{2}{c}{ trays 37 and 60} \\
\hline 3.29 & -2.29 & 1.19 & -0.19 & 1.30 & -0.30 \\
-2.29 & 3.29 & -0.19 & 1.19 & -0.30 & 1.30 \\
\hline
\end{tabular}

Finally, the sensitivity of the concentrations of the impurities in the condenser and the reboiler to the tray temperatures are calculated (cf. Table 2). The impurities that have to be kept small by the controller are impurity 1 in the condenser and impurity 2 in the reboiler. In both cases the sensitivity of the concentration to the temperature is larger on the fourth tray than on the $37^{\text {th }}$ tray. Thus, the temperatures on the trays 4 and 60 are selected as controlled variables.

Table 2. Sensitivities of the concentration of the impurities in the reboiler and the condenser to tray temperatures.

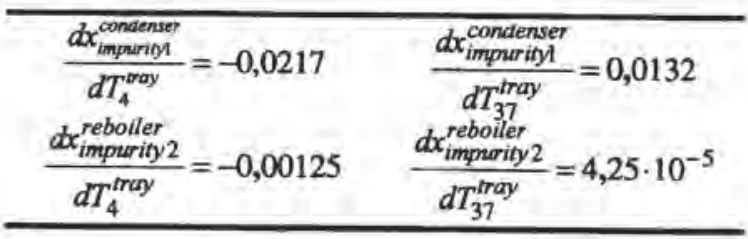

The selection of manipulated variables corresponds to the L/DV structure for distillation columns (cf. Shinskey, 1984). Other possible selections of manipulated variables (like the $\mathrm{LV}$ or the $\mathrm{DB}$ configuration) are not considered here, because the analysis above shows that the LDV structure is a good choice (RGA diagonal element close to unity, small RGA-number over the entire frequency range, small minimized condition number).

\section{LINEAR CONTROLLER DESIGN}

After the control structure is fixed, the controller can be designed. The MIMO controller design method used here is a frequency response approximation technique developed by Engell and Müller (1993). During the design procedure the desired closed loop behavior has to be specified. In this case the specification was an overshooting of maximal $10 \%$ for both controlled variables and decoupling between the loops. The remaining degrees of freedom are the rise times (and therefore the bandwidth) and the orders of the transfer functions $c_{i j}(s)$ of the controllers. With this method plant models that are unstable or contain rHP transmission zeros can be handled. However none of the above is the case for the plant model under consideration.

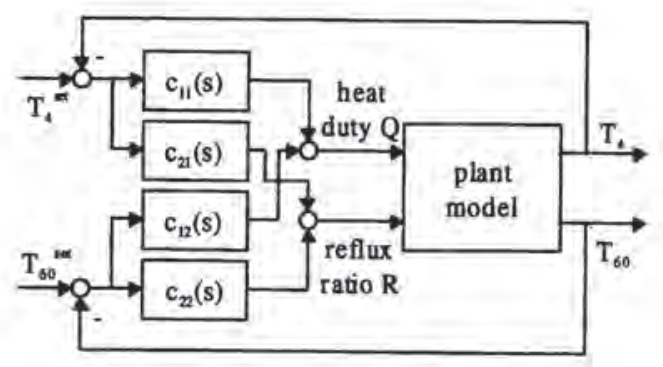

Fig. 7. Scheme of the controller structure.

The control structure is depicted in Fig. 7. The design procedure now calculates the transfer functions of the controllers $c_{i j}(s)$ for the given specifications of the rise times, the maximal overshootings and the orders of the transfer functions.

The robustness of the closed loop is observed by checking the $\mu$-conditions. For the sake of brevity the results of the $\mu$-conditions are only summarized for the different controllers. The model uncertainties that are considered here are uncorrelated delays in the manipulated variables that can be described by first order delays with rise times of $5 \mathrm{~min}$. in the reflux ratio and $20 \mathrm{~min}$. in the heat duty.

The task of the design is to find the controllers $c_{i j}(s)$ that give the highest bandwidth, observe the bounds on the manipulated variables and are robust against model uncertainties. The bounds on both manipulated variables are maximal deviations of \pm 5 from their nominal values. Since robustness and high bandwidth are counteracting specifications, a controller is calculated that observes the bounds on the controlled variables and checked for robustness afterwards. If the $\mu$-conditions are violated the bandwidth is decreased until robustness can be ensured. Another important aspect is the influence of the orders of the controllers $c_{i j}(s)$ on the performance and the robustness. One might favor PI-controllers to make the implementation in a standard PCS as easy as possible. 
In a first step the maximal achievable bandwidth of the closed loop can be estimated form the dynamics of the plant and the bounds on the manipulated variables (Müller, 1996). The calculation shows that the minimal rise time for the temperatures are about 20 $\min$. for $T_{4}$ and $1 \mathrm{~min}$. for $\mathrm{T}_{60}$. These values are much smaller than the rise times of the open loop responses of about $5 \mathrm{~h}$ for $\mathrm{T}_{4}$ and $40 \mathrm{~min}$. for $\mathrm{T}_{60}$. Thus, the bounds on the manipulated variables allow to make the closed loop much faster than the open loop.

The first controller is now calculated specifying rise times of 20 and $6 \mathrm{~min}$. The desired closed loop behavior cannot be achieved with simple PI controllers but with $3^{\text {rd }}$ order transfer functions for the $c_{i j}(s)$. The examination of the $\mu$-conditions shows, that the nominal stability, nominal performance and robust stability can be ensured but the condition for robust performance is violated. Further, omitting of one or both off-diagonal elements $c_{12}(s)$ and $c_{21}(s)$ does not result in a controller that fulfills the robust performance condition. Thus, the robustness limits the achievable bandwidth rather than the bounds on the manipulated variables in the linear case.

In the sequel different rise times for both loops were specified. The highest bandwidths for that robustness can be guaranteed, were found for rise times of $4 \mathrm{~h}$. for $T_{4}$ and of $1 \mathrm{~h}$ for $T_{60}$. Using controllers of $3^{\text {rd }}$ order for all $c_{i j}(s)$ results in a complete decoupling for the nominal linear model. If the delays in the manipulated variables are considered in a simulation study, the overshooting is slightly higher (about $15 \%$ in both loops) and a coupling between the loops of about $5 \%$ occurs. In order to reduce the effort to implement the controller, standard PI-controllers are calculated for the specifications above. The robustness analysis for the calculated PI controllers shows, that the robust performance condition is violated. Thus the reduction in the complexity of the controller leads to a less robust performance.

The next step is to check, if the robustness can be increased using only the controllers $c_{11}(s)$ and $c_{22}(s)$. This will obviously result in a stronger coupling between the two loops because the interactions cannot be compensated by the off-diagonal controller elements. The analysis for the diagonal PI-controller shows that all $\mu$-conditions are satisfied. The closed loop behavior shows the desired rise times and overshootings for the nominal model and for the case when the delays are considered. The price for the increased robustness is a coupling of about $15 \%$ in the step responses for the loops. Using higher order controllers cannot circumvent the coupling. While the robust performance condition is close to its bound for the high order controller (see above), the diagonal PI controller shows an additional margin. This margin indicates that the bandwidth can be further increased in a next step.
Subsequently the rise times were reduced in several steps and new diagonal PI-controllers were calculated. The checking of the $\mu$-conditions shows that the rise times can be reduced down to $2 \mathrm{~h}$ for $\mathrm{T}_{4}$ and $0.5 \mathrm{~h}$ for $\mathrm{T}_{60}$. The reduction in the rise times leads to an increased coupling of the loops. A maximal coupling of about $25 \%$ occurs for a step in $\mathrm{T}_{4}$ for the case that the delays are considered. The last step during controller design is to check if an additional PI element $c_{12}(s)$ or $c_{21}(s)$ can reduce the coupling while keeping the robustness properties. Using the $c_{12}$ element leads to a controller that violates the robust performance condition. On the other hand the extra $\mathrm{c}_{21}(\mathrm{~s})$ element reduces the coupling down to about $15 \%$ and fulfills all $\mu$-conditions at the same time.

\section{NONLINEAR SIMULATION}

The diagonal (controller1) and the diagonal controller with an extra element $c_{21}(s)$ (controller2) show good results regarding the bandwidth and the robustness. Thus, both controllers are tested using the nonlinear plant model. The delays in the manipulated variables are included into the nonlinear model to check the robustness. The transients after a step in the setpoint of $T_{4}$ in Fig. 8 shows that the linear and the nonlinear closed loop simulations behave similarly. The small deviations between the linear and the nonlinear loops indicate the nonlinear behavior of the process. The use of controller 2 reduces the coupling between the two loops in the linear as well as in the nonlinear case. The disturbance rejection properties of both controllers for a change in the feed flow rate of $+10 \%$ are shown in Fig. 9 and Fig. 10. Both controllers reject the effects of the disturbance on the temperatures and the concentrations of the impurities efficiently. Since both controllers are stable, robust, observe the bounds on the manipulated variables, have maximum bandwidth and reject the effect of disturbances on the concentrations of impurities all specification are met. The nonlinear behavior of the process is investigated in more detail in Grüner et al. (2001)
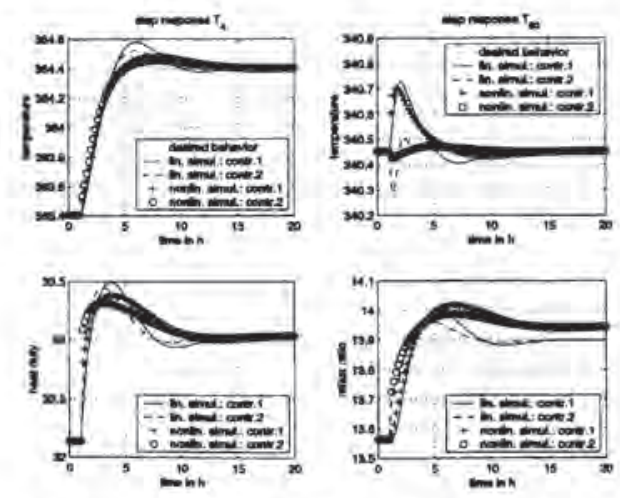

Fig. 8. Linear and nonlinear simulation of the setpoint tracking for a step in the temperature $T_{4}$. 

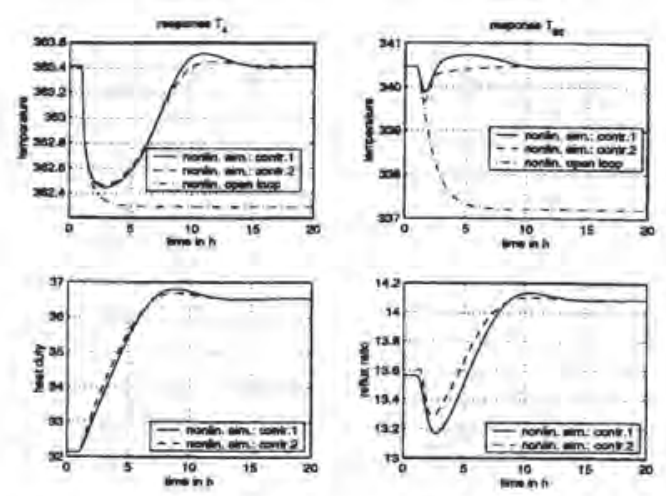

Fig. 9. Nonlinear simulation of the temperatures $T_{4}$ and $T_{60}$ for an increase of $10 \%$ in the feed flow rate.
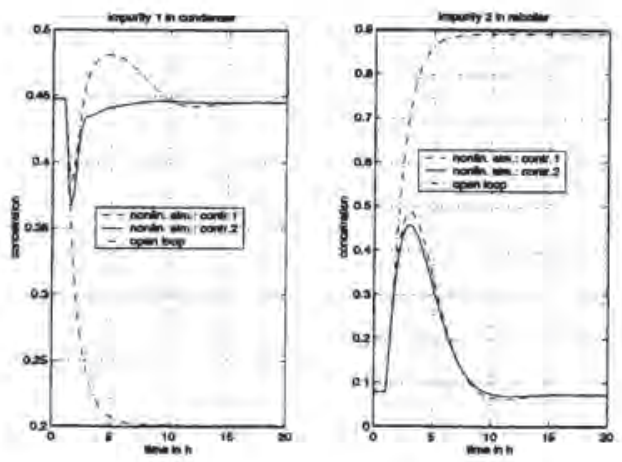

Fig. 10. Nonlinear simulation of the impurity concentrations in the condenser and the reboiler for an increase of $10 \%$ in the feed flow rate.

\section{SUMMARY}

The aim of this paper was to demonstrate the use of advanced linear controller design methods for an industrial scale reactive distillation column. Using sensitivity analysis and RGA techniques resulted in a selection of an appropriate control structure. Choosing a good control structure avoids problems that might occur later during the controller design procedure and thus allows to design a high performance controller afterwards. Advanced controller design methods combined with state-of-the-art techniques to investigate robustness were successfully applied to calculate a linear MIMO controller with good robustness and performance. Moreover, the design method used allows to fix the order (e.g. PI) and the structure (e.g. diagonal controller) of the MIMO controller. Comparing controllers of different structures and different orders facilitated to rate if the use of more complex controllers improves robustness and performance. For the reactive distillation column considered here it was shown that using simple PIcontrollers in the MIMO structure is sufficient. In the problem considered here using more complex linear controllers does not circumvent the problem that a faster performance results in poorer robustness.
The example showed that advanced linear controller design methods support the process engineer during the design procedure to find a suitable controller structure, to calculate a high performance controller and to asses the results of the design. Thus, besides improving the controller performance one of the main benefits of the methods used here is that they allow synthesizing and analyzing the controller at the same time helping to choose the most suitable controller.

\section{ACKNOWLEDGEMENT}

This research reported here was financially supported by the Bundesministerium für Bildung und Forschung (BMBF) grant no. 03C0268C/7. This support is gratefully acknowledged.

\section{REFERENCES}

Engell, S., Müller, R. (1993). Proceedings of the $2^{\text {nd }}$ European Control Conference ECC2, Groningen, 1715-1720.

Fernholz, G, Wang, W., Engell, S., Fougner, K., Bredehöft, J.-P. (2000/1999?). Proc. 1999 IEEEInternational Conference on Control Applications, 22.-26. August 1999, Hawaii, USA, 397-402.

Gehrke, V. , Reder, C., Marquardt, W. (1998).Project report LPT-pro-1998-02, RWTH Aachen .

Grüner, S., Mohl, K.-D., Kienle, A., Gilles, E.-D., Fernholz, G, Friedrich, M. (2001). Nonlinear control of an industrial reactive distillation column. Paper submitted for the DYCOPS-6, 03.0606.062001 , Korea.

Kienle, A., Lauschke, G, Gehrke, V., Gilles, E. D. (1995), Chem. Eng. Sci., 50(15), 2361-2375.

Kienle (2000a), Chem. Eng. Sci. 55(10), 1817-1825.

Kienle (2000b) Proceedings SPC-2000, Ploiesti, Romania, 72-78.

Luyben, W. L. (1992). Practical distillation control. Van Nostrand Reinhold, New York.

Mohl, K. D., Kienle, A., Gilles, E. D., Rapmund, P., Sundmacher, K., Hoffmann, U. (1999) Chem. Eng. Sci. ,54(8), 1029-1043.

Moore, C. (1992). In: Practical distillation control (W.L. Luyben. (Ed)), 140-191, Van Nostrand Reinhold, New York.

Müller, R. (1996). Entwurf von Mehrgrößenreglern durch Frequenzgang-Approximation. $\mathrm{PhD}$ thesis, University of Dortmund, Shacker Verlag, Aachen.

Shinskey, F. G (1984). Distillation Control. $2^{\text {nd }}$ edition. McGraw-Hill, New York.

Skogestad, S., Postlethwaite, I. (1996). Multivariable feedback control. Analysis and design. John Wiley \& Sons, Chichester.

Skogestad, S. (1997), Modeling, identification and control, 18, 177-217.

Skogestad, S., Morari, M. (1987). I. Chem. E Symposium Series, 104, A71-A86. 